\title{
EVOLUTION SEMIGROUPS FOR NONAUTONOMOUS CAUCHY PROBLEMS
}

\author{
GREGOR NICKEL
}

\begin{abstract}
In this paper, we characterize wellposedness of nonautonomous, linear Cauchy problems

$$
(N C P)\left\{\begin{array}{l}
\dot{u}(t)=A(t) u(t) \\
u(s)=x \in X
\end{array}\right.
$$

on a Banach space $X$ by the existence of certain evolution semigroups.

Then, we use these generation results for evolution semigroups to derive wellposedness for nonautonomous Cauchy problems under some "concrete" conditions. As a typical example, we discuss the so called "parabolic" case.
\end{abstract}

\section{BASIC DEFINITIONS}

In this section, we introduce the basic definitions and notations in order to discuss nonautonomous Cauchy problems in terms of evolution families and evolution semigroups. In addition, we mention some of their fundamental properties.

The solution of a nonautonomous Cauchy problem on some Banach space $X$ can be given by a so called evolution family which can be defined as follows.

Definition 1.1 (Evolution family). A family $(U(t, s))_{t \geq s}$ of linear, bounded operators on a Banach space $X$ is called an (exponentially bounded) evolution family if

(i) $U(t, r) U(r, s)=U(t, s), U(t, t)=I d$ for all $t \geq r \geq s \in \mathbb{R}$,

(ii) the mapping $(t, s) \mapsto U(t, s)$ is strongly continuous,

(iii) $\|U(t, s)\| \leq M e^{\omega(t-s)}$ for some $M \geq 1, \omega \in \mathbb{R}$ and all $t \geq s \in \mathbb{R}$.

1991 Mathematics Subject Classification. Primary 47D06; secondary 47A55.

Key words and phrases. Evolution semigroup, nonautonomous abstract Cauchy problem, perturbation theory, parabolic problems.

Received: October 14, 1996. 
To every (exponentially bounded) evolution family we can associate $C_{0^{-}}$ semigroups on $X$-valued function spaces. These semigroups characterize the behavior of the evolution family completely and, consequently, will be called evolution semigroups. Evolution semigroups, first introduced in 1974 by Howland [5] and studied in 1976 by Evans [2], recently attracted a great deal of interest, see, e. g., [8], [9], [10], [13], [15], [16]. In particular, it was possible to characterize certain asymptotic behavior of evolution families by spectral properties of the corresponding evolution semigroup and its generator. Here, we choose the Banach space

$$
\mathcal{C}_{0}:=C_{0}(\mathbb{R}, X)=\left\{f: \mathbb{R} \rightarrow X: f \text { is continuous and } \lim _{|t| \rightarrow \infty} f(t)=0\right\},
$$

normed by $\|f\|:=\sup _{t \in \mathbb{R}}\|f(t)\|$, for $f \in \mathcal{C}_{0}$.

It is an easy exercise to show that the following definition yields a strongly continuous semigroup.

Definition 1.2 (Evolution semigroup). For every evolution family $(U(t, s))$, $t \geq s$, we define the corresponding evolution semigroup $(\mathcal{T}(t))_{t \geq 0}$ on the space $\mathcal{C}_{0}$ by

$$
(\mathcal{T}(t) f)(s):=U(s, s-t) f(s-t)
$$

for $f \in \mathcal{C}_{0}, s \in \mathbb{R}$ and $t \geq 0$. We denote its generator by $(\mathcal{G}, D(\mathcal{G}))$.

Since each operator of the above evolution semigroup is a product of a multiplication operator and a translation, it is useful to consider also the following concepts. We denote by

$$
\mathcal{C}^{1}:=\left\{f \in C^{1}(\mathbb{R}, X): f, f^{\prime} \in \mathcal{C}_{0}\right\}
$$

the domain of the generator $\left(-\frac{d}{d x}, D\left(-\frac{d}{d x}\right)\right)$ of the right translation (semi)group $(\mathcal{T} r(t))_{t \geq 0}$ on the space $\mathcal{C}_{0}$ defined by

$$
(\mathcal{T} r(t) f)(s):=f(s-t) \text { for } f \in \mathcal{C}_{0} \text { and } s \in \mathbb{R}, t \geq 0 .
$$

Thus, the evolution semigroup operators can be written as

$$
\mathcal{T}(t) f=U(\cdot, \cdot-t) \mathcal{T} r(t) f .
$$

We can recover the evolution family from the evolution semigroup by choosing a function $f \in \mathcal{C}_{0}$ with $f(s)=x$. Then we obtain, for every $s \in \mathbb{R}$ and $t \geq s, U(t, s) x=(\mathcal{T} r(s-t) \mathcal{T}(t-s) f)(s)$

Multiplication operators stem from bounded, strongly continuous operator valued functions $F \in C_{b}\left(\mathbb{R}, \mathcal{L}_{s}(X)\right)$ and are defined on the space $\mathcal{C}_{0}$ by $(F f)(s):=F(s) f(s), s \in \mathbb{R}$, (cf. [4]). In particular, for every scalar function $\phi \in C_{b}(\mathbb{R})$ we obtain a multiplication operator $M_{\phi}$ by $\left(M_{\phi} f\right)(s):=\phi(s) f(s), \quad s \in \mathbb{R}$. 
For a family $(A(t), D(A(t)))_{t \in \mathbb{R}}$ of unbounded operators on $X$ we consider the corresponding multiplication operator $(A(\cdot), D(A(\cdot)))$ on the space $\mathcal{C}_{0}$ defined by

$$
\begin{aligned}
D(A(\cdot)):= & \left\{f \in \mathcal{C}_{0}: f(s) \in D(A(s))\right. \\
& \text { for all } \left.s \in \mathbb{R}, \text { and } s \mapsto A(s) f(s) \in \mathcal{C}_{0}\right\}, \\
(A(\cdot) f)(s):= & A(s) f(s) \text { for all } s \in \mathbb{R} .
\end{aligned}
$$

In the subsequent sections we need a characterization of evolution semigroups on the space $\mathcal{C}_{0}$. Its proof is based on ideas of Evans [2], Theorem 1.6 and Howland [5], Theorem 1. It was, later on, extended to Banach function spaces in [13], Theorem 3.4. A proof for evolution semigroups on the space $\mathcal{C}_{0}$ can be found in [18].

Proposition 1.3. Let $(\mathcal{T}(t))_{t \geq 0}$ be a $C_{0}$-semigroup with generator $(\mathcal{G}, D(\mathcal{G}))$ on the Banach space $\mathcal{C}_{0}$. Then the following assertions are equivalent.

(i) The semigroup $(\mathcal{T}(t))_{t \geq 0}$ is an evolution semigroup, i. e., there exists an evolution family $(U(t, s))_{t \geq s}$ on the space $X$ such that $\mathcal{T}(t)=$ $U(\cdot, \cdot-t) \mathcal{T} r(t)$.

(ii) For all $\phi \in C_{0}(\mathbb{R}), f \in \mathcal{C}_{0}$ we have $(\mathcal{T}(t) \phi f)(s)=\phi(s-t)(\mathcal{T}(t) f)(s), \quad s \in \mathbb{R}, \quad t \geq 0$.

(iii) There exists a core $\mathcal{D}$ for $\mathcal{G}$ such that for all $\phi \in C_{c}^{1}(\mathbb{R}), f \in \mathcal{D}$ we have $\phi f \in D(\mathcal{G})$ and $\mathcal{G} \phi f=-\phi^{\prime} f+\phi \mathcal{G} f$.

For later use, we add a characterization for unbounded multiplication operators on $\mathcal{C}_{0}$.

Lemma 1.4. For a linear operator $(\mathcal{A}, D(\mathcal{A}))$ on the space $\mathcal{C}_{0}$ the following statements are equivalent.

(i) The operator $(\mathcal{A}, D(\mathcal{A})) \subseteq(\mathcal{A}(\cdot), D(\mathcal{A}(\cdot)))$ is contained in a multiplication operator given by a family of linear operators $(\mathcal{A}(t), D(\mathcal{A}(t)))_{t \in \mathbb{R}}$ on $X$.

(ii) For all $f \in D(\mathcal{A})$ with $f(s)=0$ we have $(\mathcal{A} f)(s)=0$.

Proof. If $\mathcal{A}$ is a multiplication operator and $f \in D(\mathcal{A})$ with $f(s)=0$ we clearly have $(\mathcal{A} f)(s)=\mathcal{A}(s) f(s)=0$.

Conversely, if property (ii) holds we define for every $t \in \mathbb{R}$ a linear operator $(\mathcal{A}(t), D(\mathcal{A}(t)))$ on $X$ by

$$
\begin{aligned}
D(\mathcal{A}(t)) & :=\left\{x \in X: \text { there exists } f_{x} \in D(\mathcal{A}) \text { with } f_{x}(t)=x\right\}, \\
\mathcal{A}(t) x & :=\left(\mathcal{A} f_{x}\right)(t) \quad \text { for all } x \in D(\mathcal{A}(t)) .
\end{aligned}
$$

By using property (ii) we obtain a well-defined linear operator $(\mathcal{A}(t), D(\mathcal{A}(t)))$ on $X$ for every $t \in \mathbb{R}$ and we see easily that $(\mathcal{A}, D(\mathcal{A})) \subseteq$ $(\mathcal{A}(\cdot), D(\mathcal{A}(\cdot)))$. 
2. Wellposedness of nonautonomous Cauchy problems - AN ABSTRACT CHARACTERIZATION

In the first part of this section we define our concept of wellposedness for nonautonomous Cauchy problems

$$
(N C P)_{s, x}\left\{\begin{array}{l}
\dot{u}(t)=A(t) u(t) \\
u(s)=x
\end{array}\right.
$$

for $t \geq s \in \mathbb{R}$, where $(A(t), D(A(t)))_{t \in \mathbb{R}}$ is family of linear operators on some Banach space $X$. If the Cauchy problem is considered for all initial times $s \in \mathbb{R}$ we denote it by $(N C P)$.

Definition 2.1. A continuous function $u:[s, \infty) \rightarrow X$ is called

(i) classical solution of $(N C P)_{s, x}$ if $u \in C^{1}((s, \infty), X), u(t) \in D(A(t))$ for all $t>s, u(s)=x$, and $\dot{u}(t)=A(t) u(t)$ for $t>s$,

(ii) (strict) solution of $(N C P)_{s, x}$ if $u \in C^{1}([s, \infty), X), u(t) \in D(A(t))$ for all $t \geq s, u(s)=x$, and $\dot{u}(t)=A(t) u(t)$ for $t \geq s$.

By examples, it can be shown that the spaces $Y_{s} \subseteq X$ of initial values with differentiable solution of $(N C P)_{s, x}$ may vary dramatically with $s \in \mathbb{R}$ (see [3]) and that it is useful to differ between these spaces and the domains of the righthand side operators $D(A(s))$ ([11], Example 3.5).

So, the following slight modification of Kellermann's definition, [6], Definition 1.1, seems to be an appropriate definition of wellposedness.

Definition 2.2 (Wellposedness). For a family $(A(t), D(A(t)))_{t \in \mathbb{R}}$ of linear operators on the Banach space $X$ the nonautonomous Cauchy problem $(N C P)$ is called wellposed with regularity subspaces $\left(Y_{s}\right)_{s \in \mathbb{R}}$ if

(i) (Existence) For all $s \in \mathbb{R}$ the subspace

$$
\begin{aligned}
Y_{s} & :=\left\{y \in X: \text { there exists a strict solution for }(N C P)_{s, y}\right\} \\
& \subset D(A(s))
\end{aligned}
$$

is dense in $X$.

(ii) (Uniqueness) For every $y \in Y_{s}$ the solution $u_{s}(\cdot, y)$ is unique.

(iii) (Continuous dependence) The solution depends continuously on $s$ and $y, i$. e., if $s_{n} \rightarrow s \in \mathbb{R}, y_{n} \rightarrow y \in Y_{s}$ with $y_{n} \in Y_{s_{n}}$ then we have $\left\|\hat{u}_{s_{n}}\left(t, y_{n}\right)-\hat{u}_{s}(t, y)\right\| \rightarrow 0$ uniformly for $t$ in compact subsets of $\mathbb{R}$, where

$$
\hat{u}_{r}(t, y):=\left\{\begin{aligned}
u_{r}(t, y) & \text { if } r \leq t \\
y & \text { if } r>t .
\end{aligned}\right.
$$

If, in addition, there exist constants $M \geq 1$ and $\omega \in \mathbb{R}$ such that

$$
\left\|u_{s}(t, y)\right\| \leq M e^{\omega(t-s)}\|y\|
$$

for all $y \in Y_{s}$ and $t \geq s$ the nonautonomous Cauchy problem $(N C P)$ is called wellposed with exponentially bounded solutions. 
In the second part of this section we want to connect the Definition 2.2 of wellposedness to the existence of an evolution family (cf. Definition 1.1) solving the nonautonomous Cauchy problem. In general, however, and in contrast to the behavior of $C_{0}$-semigroups (the autonomous case), the algebraic properties of an evolution family do not imply any differentiability on a dense subspace. This already occurs in one dimension (cf. [9], Counterexample 2.3).

Example 2.3. Take a continuous function $p: \mathbb{R} \rightarrow[1,2]$ and define $U(t, s)$ $:=\frac{p(t)}{p(s)}$ for $s \leq t$. Then $(U(t, s))_{t \geq s}$ is a (uniformly) continuous evolution family on $\mathbb{C}$ which is not differentiable if we choose the function $p$ nowhere differentiable.

So we have to add some differentiability assumptions in order to solve a nonautonomous Cauchy problem by an evolution family.

Definition 2.4 (EVF solving (NCP)). An evolution family $(U(t, s))_{t \geq s}$ is called an evolution family solving $(N C P)$, if for every $s \in \mathbb{R}$ the regularity subspace

$$
Y_{s}:=\left\{y \in X:[s, \infty) \ni t \mapsto U(t, s) y \text { solves }(N C P)_{s, y}\right\}
$$

is dense in $X$.

Now we can characterize wellposedness of a nonautonomous Cauchy problem by the existence of a unique solving evolution family (see [6], Proposition 1.4). A proof can be found in [11], Proposition 3.10.

Proposition 2.5. Let $X$ be a Banach space, $(A(t), D(A(t)))_{t \in \mathbb{R}}$ a family of linear operators on $X$ and consider the nonautonomous Cauchy problem $(N C P)$.

The following assertions are equivalent.

(i) The nonautonomous Cauchy problem $(N C P)$ is wellposed.

(ii) There exists a unique evolution family $(U(t, s))_{t \geq s}$ solving $(N C P)$.

Corollary 2.6. The nonautonomous Cauchy problem is wellposed with exponentially bounded solutions if and only if there exists a unique exponentially bounded evolution family solving it.

In the last part of this section, the point of departure is a strongly continuous evolution family $(U(t, s))_{t \geq s}$ and the corresponding evolution semigroup $(\mathcal{T}(t))_{t \geq 0}$. In general, no differentiability holds for the evolution family, but the evolution semigroup is always differentiable on the domain of its generator. The differentiability of the evolution family, i. e. wellposedness of a corresponding $(N C P)$, will now be characterized by some properties of the generator $(\mathcal{G}, D(\mathcal{G}))$ of $(\mathcal{T}(t))_{t \geq 0}$. 
Let $(U(t, s))_{t>s}$ be an evolution family, $(\mathcal{T}(t))_{t>0}$ the corresponding evolution semigroup with generator $(\mathcal{G}, D(\mathcal{G}))$ on the space $\mathcal{C}_{0}:=C_{0}(\mathbb{R}, X)$. On this space we define the operator $(\mathcal{A}, D(\mathcal{A}))$ by

$$
\begin{aligned}
D(\mathcal{A}) & :=\left\{f \in \mathcal{C}_{0}: \lim _{t \searrow 0} \frac{[U(\cdot, \cdot-t)-I d]}{t} f(\cdot) \in \mathcal{C}_{0}\right\}, \\
\mathcal{A} f & :=s \mapsto \lim _{t \searrow 0} \frac{[U(s, s-t)-I d]}{t} f(s) .
\end{aligned}
$$

From the definition and Lemma 1.4 we see that $(\mathcal{A}, D(\mathcal{A}))$ is contained in a multiplication operator on $\mathcal{C}_{0}$. We denote its extension again by $\mathcal{A}=\mathcal{A}(\cdot)$. For this multiplication operator we also consider the operators $(\mathcal{A}(t), D(\mathcal{A}(t)))$ defined as in Lemma 1.4 by

$$
\begin{aligned}
D(\mathcal{A}(t)) & :=\left\{x \in X: \exists f_{x} \in D(\mathcal{A}) \text { with } f_{x}(t)=x\right\} \\
\mathcal{A}(t) x & :=\left(\mathcal{A} f_{x}\right)(t) .
\end{aligned}
$$

As a first step towards a characterization of wellposedness we give a connection between the spaces $\mathcal{C}^{1}, D(\mathcal{G})$, and $D(\mathcal{A})$. Remark that the space $\Delta$ defined below can be zero (cf. Example 2.3).

Proposition 2.7. With the notation above we have

$$
\Delta:=\mathcal{C}^{1} \cap D(\mathcal{A})=\mathcal{C}^{1} \cap D(\mathcal{G})=D(\mathcal{G}) \cap D(\mathcal{A}),
$$

and

$$
\mathcal{G} f=\mathcal{A} f-f^{\prime} \quad \text { for } f \in \Delta .
$$

Moreover, for $f \in \Delta$, the differential equation

$$
-\frac{\partial}{\partial s} U(t, s) f(s)=U(t, s)\left[\mathcal{A}(s) f(s)-f^{\prime}(s)\right]
$$

holds for all $t \geq s \in \mathbb{R}$ with

$$
\lim _{|s| \rightarrow \infty}\left\|\mathcal{A}(s) f(s)-f^{\prime}(s)\right\|=0 .
$$

Proof. For $f \in \mathcal{C}_{0}$ and $t>0$ we consider the identity

$$
\begin{aligned}
\underbrace{\frac{1}{t}(\mathcal{T}(t) f-f)}_{I} & =\frac{1}{t}(U(\cdot, \cdot-t) f(\cdot-t)-f(\cdot)) \\
& =\underbrace{U(\cdot, \cdot-t) \frac{f(\cdot-t)-f(\cdot)}{t}}_{I I}+\underbrace{\frac{U(\cdot, \cdot-t)-I d}{t} f(\cdot)}_{I I I} .
\end{aligned}
$$


By definition, expression $I$ converges - for $t \searrow 0$ - to $\mathcal{G} f$ for $f \in D(\mathcal{G})$, expression $I I$ converges to $f^{\prime}$ for $f \in \mathcal{C}^{1}$ and, finally, expression $I I I$ converges to $\mathcal{A} f$ for $f \in D(\mathcal{A})$. Therefore equation (2.6) gives the implications

$$
\begin{aligned}
f \in \mathcal{C}^{1} \cap D(\mathcal{A}) & \Rightarrow f \in D(\mathcal{G}), \\
f \in \mathcal{C}^{1} \cap D(\mathcal{G}) & \Rightarrow f \in D(\mathcal{A}), \\
f \in D(\mathcal{G}) \cap D(\mathcal{A}) & \Rightarrow f \in \mathcal{C}^{1},
\end{aligned}
$$

and shows equation (2.3). To justify the last implication we remark that $f \in D(\mathcal{A})$ implies

$$
\begin{aligned}
& \lim _{t \searrow 0}\left[U(\cdot, \cdot-t) \frac{f(\cdot-t)-f(\cdot)}{t}-\frac{f(\cdot-t)-f(\cdot)}{t}\right] \\
& \quad=\lim _{t \searrow 0}\left[\frac{U(\cdot, \cdot-t)-I d}{t} f(\cdot-t)-\frac{U(\cdot, \cdot-t)-I d}{t} f(\cdot)\right]=0 .
\end{aligned}
$$

Thus, for $f \in \Delta$ we obtain

$$
\frac{d}{d t} \mathcal{T}(t) f=\mathcal{T}(t) \mathcal{G} f=\mathcal{T}(t)\left(\mathcal{A} f-f^{\prime}\right) \quad \text { for all } t \geq 0 .
$$

Explicitly this means that

$$
\frac{\partial}{\partial t}[U(s, s-t) f(s-t)]=U(s, s-t)\left[\mathcal{A}(s-t) f(s-t)-f^{\prime}(s-t)\right]
$$

holds in the space $\mathcal{C}_{0}$. Therefore the differential equation (2.4) follows for all $t \geq s$. The property $(2.5)$ simply reflects that $\mathcal{G} f \in \mathcal{C}_{0}$.

If we now assume that the orbit of $f \in \Delta$ under $(\mathcal{T}(t))_{t \geq 0}$ remains in $\Delta$, we arrive at the correct differential equation.

Proposition 2.8. With the notation above the following assertions for a function $f \in \mathcal{C}_{0}$ are equivalent.

(i) We have $T(t) f \in \Delta=\mathcal{C}^{1} \cap D(\mathcal{G})$ for all $t \geq 0$.

(ii) For the function $f \in \mathcal{C}^{1}$ we have $U(t, s) f(s) \in D(\mathcal{A}(t))$ for all $t \geq s$ and the differential equation

$$
\frac{\partial}{\partial t} U(t, s) f(s)=\mathcal{A}(t) U(t, s) f(s)
$$

holds for all $t \geq s$. Moreover, we have

$$
\mathcal{A}(\cdot) U(\cdot, \cdot-t) f(\cdot-t) \in \mathcal{C}_{0}
$$

for all $t \geq 0$.

Proof. For $\mathcal{T}(t) f \in \mathcal{C}^{1} \cap D(\mathcal{G})$ we obtain $\mathcal{T}(t) f \in D(\mathcal{A})$ by Proposition 2.7 and

$$
\mathcal{A T}(t) f=\mathcal{G} \mathcal{T}(t) f+(\mathcal{T}(t) f)^{\prime}=\frac{\partial}{\partial t} \mathcal{T}(t) f+(\mathcal{T}(t) f)^{\prime}
$$


For the sake of simplicity, we argue in the following with differential quotients instead of using difference quotients. For the two terms of (2.9) we obtain (at least formally)

$$
\begin{aligned}
\left(\frac{\partial}{\partial t} \mathcal{T}(t) f\right)(s) & =\frac{\partial}{\partial t} U(s, s-t) f(s-t) \\
& =-\left.\frac{\partial}{\partial x} U(s, x)\right|_{x=s-t} f(s-t)-U(s, s-t) f^{\prime}(s-t)
\end{aligned}
$$

and

$$
\begin{aligned}
(\mathcal{T}(t) f)^{\prime}= & \frac{\partial}{\partial s} U(s, s-t) f(s-t) \\
= & \left.\frac{\partial}{\partial x} U(x, s-t)\right|_{x=s} f(s-t)+\left.\frac{\partial}{\partial x} U(s, x)\right|_{x=s-t} f(s-t) \\
& +U(s, s-t) f^{\prime}(s-t) .
\end{aligned}
$$

Taking the sum of (2.10) and (2.11) we obtain that $\left.\frac{\partial}{\partial x} U(x, s-t)\right|_{x=s} f(s-t)$ is well defined and

$$
(\mathcal{A T}(t) f)(s)=\mathcal{A}(s) U(s, s-t) f(s-t)=\left.\frac{\partial}{\partial x} U(x, s-t)\right|_{x=s} f(s-t)
$$

holds in the space $\mathcal{C}_{0}$. Thus the differential equation (2.7) holds for all $t \geq s$ and property (2.8) follows.

Conversely, if we suppose (2.7) and the property (2.8) we see by equation (2.12) that $\mathcal{T}(t) f \in D(\mathcal{A})$ for $t \geq 0$. Thus, by Proposition 2.7 we obtain $f \in D(\mathcal{G})$, hence $\mathcal{T}(t) f \in D(\mathcal{G})$ for all $t \geq 0$ and, finally, it results $T(t) f \in \Delta=\mathcal{C}^{1} \cap D(\mathcal{G})$ for all $t \geq 0$.

As the main result of this section we now relate properties of the domain $D(\mathcal{G})$ to wellposedness of the nonautonomous Cauchy problem $(N C P)$. Roughly speaking $(N C P)$ is wellposed if there are sufficiently many orbits for $(\mathcal{T}(t))_{t \geq 0}$ staying in the space of differentiable functions $\mathcal{C}^{1}$.

Theorem 2.9. Let $X$ be a Banach space and $\left(A(t), D(A(t))_{t \in \mathbb{R}}\right.$ a family of linear operators on $X$. The following assertions are equivalent.

(i) The nonautonomous Cauchy problem $(N C P)$ for the family $(A(t))_{t \in \mathbb{R}}$ is wellposed (with exponentially bounded solutions).

(ii) There exists a unique evolution semigroup $(\mathcal{T}(t))_{t \geq 0}$ with generator $(\mathcal{G}, D(\mathcal{G}))$ and an invariant core $\mathcal{D} \subseteq \Delta=\mathcal{C}^{1} \cap D(\mathcal{G})$ such that

$$
\mathcal{G} f+f^{\prime}=\mathcal{A}(\cdot) f=A(\cdot) f
$$

for $f \in \mathcal{D}$.

Proof. (i) $\Rightarrow$ (ii). If $(N C P)$ is wellposed (with exponentially bounded solutions) there exists by Proposition 2.5 a unique solving evolution family 
$(U(t, s))_{t \geq s}$, and therefore also a (unique) evolution semigroup $(\mathcal{T}(t))_{t \geq 0}$. We only need to find an invariant core for $(\mathcal{T}(t))_{t \geq 0}$ with the claimed properties. It is obtained using an idea from [7], Proposition 2.9. Thus, consider $s \in \mathbb{R}$, $y \in Y_{s}$ and a function $\alpha \in C_{c}^{\infty}(\mathbb{R})$ - the space of smooth functions with compact support - with supp $\alpha \subset[s, \infty)$. Then the function $f \in \mathcal{C}_{0}$ defined by

$$
f(t):= \begin{cases}\alpha(t) U(t, s) y & \text { if } t>s \\ 0 & \text { otherwise }\end{cases}
$$

is contained in $D(\mathcal{G})$. Moreover, we have $f \in \Delta$ and $\mathcal{G} f=-f^{\prime}+A(\cdot) f$. This can be seen by calculating $\mathcal{T}(t) f$ as

$$
\mathcal{T}(t) f(r)= \begin{cases}\alpha(r-t) U(r, s) y & \text { if } r-t>s \\ 0 & \text { otherwise }\end{cases}
$$

which implies

$$
(\mathcal{G} f)(r)=\left.\frac{d}{d t} \mathcal{T}(t) f(r)\right|_{t=0}=-\alpha^{\prime}(r) U(r, s) y .
$$

Since $U(t, s)$ solves $(N C P)$ we have

$$
\begin{aligned}
\frac{d}{d r} f(r) & =\alpha^{\prime}(r) U(r, s) y+\alpha(r) \frac{\partial}{\partial r} U(r, s) y \\
& =\alpha^{\prime}(r) U(r, s) y+\alpha(r) A(r) U(r, s) y .
\end{aligned}
$$

Combining equation (2.14) and (2.15) we end up with

$$
\mathcal{G} f=\mathcal{A}(\cdot) f-f^{\prime}=A(\cdot) f-f^{\prime} .
$$

It remains to show that the space

$$
\begin{aligned}
\mathcal{D}:= & \operatorname{span}\left\{\alpha(t) U(t, s) y: s \in \mathbb{R}, y \in Y_{s}, \alpha \in C_{c}^{\infty}(\mathbb{R})\right. \\
& \text { with } \operatorname{supp} \alpha \subset[s, \infty)\}
\end{aligned}
$$

is invariant under $(\mathcal{T}(t))_{t \geq 0}$ and dense in $\mathcal{C}_{0}$. From equation $(2.13)$ we immediately see the invariance. For the proof of the denseness we refer to [7], Proposition 2.9.

Now uniqueness of an evolution semigroup with the above properties follows by a standard argument. One only has to consider the derivative of the function $s \mapsto \mathcal{S}(t-s) \mathcal{T}(s) f$ for two such evolution semigroups and $f \in \mathcal{D}$.

(ii) $\Rightarrow$ (i). Conversely, suppose that there exists a unique evolution semigroup and thus a unique evolution family $(U(t, s))_{t \geq s}$ and an invariant core $\mathcal{D} \subseteq \Delta \subset \mathcal{C}_{0}$ with

$$
\mathcal{G} f+f^{\prime}=\mathcal{A}(\cdot) f=A(\cdot) f
$$

for $f \in \mathcal{D}$. Then we obtain dense subspaces

$$
Y_{s}:=\{y \in X: \exists f \in \mathcal{D} \text { with } f(s)=y\} \subseteq X
$$


for all $s \in \mathbb{R}$ and by Proposition 2.8 for $y \in Y_{s}$ the unique solution of $(N C P)$ is given by $t \mapsto U(t, s) y$.

By similar reasoning we obtain the following result.

Proposition 2.10 (Parabolic case). Consider for an evolution semigroup $(\mathcal{T}(t))_{t \geq 0}$ on the space $\mathcal{C}_{0}$ the condition $\mathcal{T}(t)\left[\mathcal{C}_{0}\right] \subset D(\mathcal{A})$ for $t>0$. Then we have $\mathcal{T}(t)[D(\mathcal{G})] \subset \Delta$ for $t>0$, and the differential equation

$$
\frac{\partial}{\partial t} U(t, s) x=\mathcal{A}(t) U(t, s) x
$$

holds for all $t>s$ and for all $x \in X$.

\section{Application to parabolic Conditions}

The generator $(\mathcal{G}, D(\mathcal{G}))$ of an evolution semigroup is formally given as a sum $\mathcal{G}=\left[A(\cdot)-\frac{d}{d x}\right]$ of an operator valued multiplication operator $A(\cdot)$ and the differential operator $-\frac{d}{d x}$. Thus, if we find conditions on the family of operators $(A(t))_{t \in \mathbb{R}}$ which enable us to define the above formal sum yielding a generator of a $C_{0}$-semigroup on $\mathcal{C}_{0}$ we can obtain wellposedness results.

The known conditions implying wellposedness are generally divided into assumptions of "parabolic" and of "hyperbolic" type. The main difference between these two types is the assumption of $A(t)$ being generators of analytic semigroups in the parabolic case, the assumption of stability for certain products in the hyperbolic case. In both cases one has to add some continuity assumption for the mapping $t \mapsto A(t)$.

In order to illustrate our approach, we use the following parabolic conditions (see [12]).

Assumption 3.1 (Parabolic case).

(P1) The domain $D:=D(A(t))$ is dense in $X$ and independent of $t \in \mathbb{R}$.

(P2) For each $t \in \mathbb{R}$ the operator $A(t)$ is the generator of an analytic semigroup. For all $t \in \mathbb{R}$, the resolvent $R(\lambda, A(t))$ exists for all $\lambda \in \mathbb{C}$ with $\Re \lambda \geq 0$ and there is a constant $M \geq 1$ such that

$$
\|R(\lambda, A(t))\| \leq \frac{M}{|\lambda|+1} \quad \text { for } \Re \lambda \geq 0, \quad t \in \mathbb{R} .
$$

(P3) There exist constants $L \geq 0$ and $0<\alpha \leq 1$ such that

$$
\left\|(A(t)-A(s)) A(0)^{-1}\right\| \leq L|t-s|^{\alpha} \text { for all } t, s \in \mathbb{R} .
$$

It is possible to prove wellposedness of $(N C P)$ under these assumptions (cf. [12], Theorem 5.6.1). In the following, we show how this wellposedness result can be obtained by evolution semigroup techniques. A corresponding approach to "hyperbolic" conditions can be found in [11], Section 4.2.

First, we will sketch the idea which is inspired by Acquistapace [1] (see also Remark 3.8 below). We consider an evolution family $(U(t, s))_{t \geq s}$ solving 
$(N C P)$ for a family $(A(t))_{t \in \mathbb{R}}$ and approximate this solution by the function $V(t, s):=e^{(t-s) A(s)}$. Consider now the function $[s, t] \ni r \mapsto U(t, r) e^{(r-s) A(s)}$. By differentiating it and integrating from $s$ to $t$ we obtain the integral equation for $(U(t, s))_{t \geq s}$

$$
U(t, s)=e^{(t-s) A(s)}+\int_{s}^{t} U(t, r)(A(r)-A(s)) e^{(r-s) A(s)} d r .
$$

We now consider equation (3.18) at $t:=x \in \mathbb{R}$ and $s:=x-\tau$ and multiply with the right translation operator $\mathcal{T} r(\tau)$. For the evolution semigroup $(\mathcal{T}(\tau))_{\tau \geq 0}$ corresponding to $(U(t, s))_{t \geq s}$, this yields the identity

$$
\begin{aligned}
\mathcal{T}(\tau)= & U(\cdot, \cdot-\tau) \mathcal{T} r(\tau) \\
= & e^{\tau A(\cdot-\tau)} \mathcal{T} r(\tau) \\
& +\int_{0}^{\tau} U(\cdot, \cdot-\tau+r)(A(\cdot-\tau+r)-A(\cdot-\tau)) e^{r A(\cdot-\tau)} d r \mathcal{T} r(\tau) \\
= & \mathcal{T} r(\tau) e^{\tau A(\cdot)}+\int_{0}^{\tau} \mathcal{T}(\tau-r)(A(\cdot)-A(\cdot-r)) \mathcal{T} r(r) e^{r A(\cdot)} d r .
\end{aligned}
$$

This is an integral equation for the evolution semigroup $(\mathcal{T}(\tau))_{\tau \geq 0}$. Solving this equation can be interpreted as solving a perturbation problem on the space $\mathcal{C}_{0}$, where the unperturbed part is the product of the two $C_{0}$-semigroups $(\mathcal{T} r(\tau))_{\tau \geq 0}$ and $\left(e^{\tau A(\cdot)}\right)_{\tau \geq 0}$.

Accordingly, we are led to perturbation theory for semigroups, and we first state a lemma which might be of independent interest.

Lemma 3.2. Let $(T(t))_{t \geq 0},(S(t))_{t \geq 0}$ be $C_{0}$-semigroups on the Banach space $X$ and denote their generators by $(\bar{A}, D(A))$ or $(B, D(B))$, respectively.

Let the domain $D(A)$ be $(S(t))$-invariant and define for every $t \geq 0$ an operator

$$
\begin{aligned}
C(t) & : \quad D(A) \rightarrow X \\
C(t) x & :=A S(t) x-S(t) A x, \quad x \in D(A) .
\end{aligned}
$$

Let the function $t \mapsto C(t) T(t) x$ be continuous for all $x \in D(A)$ and let there exist some constants $T>0$ and $\gamma<1$ such that

$$
\int_{0}^{T}\|C(\tau) T(\tau) x\| d \tau \leq \gamma\|x\|
$$

for every $x \in D(A)$. Then there exists a unique strongly continuous function $(U(t))_{t \geq 0}$ solving the integral equation

$$
U(t) x=S(t) T(t) x+\int_{0}^{t} U(t-\tau) C(\tau) T(\tau) x d \tau
$$


for all $x \in D(A)$ and $0 \leq t \leq T$. It is also given by a series expansion

$$
U(t)=\sum_{n=0}^{\infty}\left(\left(V_{A}^{B}\right)^{n} S T\right)(t)
$$

for $0 \leq t \leq T$, where $\left(\left(V_{A}^{B}\right) S T\right)(t)$ is the continuous extension of

$$
D(A) \ni x \mapsto \int_{0}^{t} S(t-\tau) T(t-\tau) C(\tau) T(\tau) x d \tau .
$$

Let now the domain $D(B)$ be $(T(t))$-invariant and let the function

$$
[0, t] \ni \tau \mapsto T(t-\tau) C(\tau) T(\tau) x
$$

be a continuous function in the space $D(B)$ for every $x \in D:=D(A) \cap D(B)$. Moreover, let $T(t) X \subset D(A)$ for $t>0$ and

$$
\begin{gathered}
\lim _{\varepsilon_{1}, \varepsilon_{2} \searrow 0} \int_{t-\varepsilon_{1}}^{t-\varepsilon_{2}} S(t-\tau) A T(t-\tau) C(\tau) T(\tau) x=0, \\
\lim _{\varepsilon_{1}, \varepsilon_{2} \searrow 0} \int_{t-\varepsilon_{1}}^{t-\varepsilon_{2}} A S(t-\tau) T(t-\tau) C(\tau) T(\tau) x=0
\end{gathered}
$$

for $x \in D$ and $t>0$. Finally, let the space $D$ be dense in $X$. Then the family $(U(t))_{0 \leq t \leq T}$ can be extended to a $C_{0}$-semigroup denoted by $(U(t))_{t \geq 0}$. For its generator $(G, D(G))$ we have

$$
D(B) \cap D(A) \subset D(G) \quad \text { and } \quad G x=A x+B x
$$

for $x \in D$. The space $D$ is an invariant core.

Proof. First Step: Denote by $\mathcal{X}:=\mathcal{C}\left([0, T], \mathcal{L}_{s}(X)\right)$ the space of all strongly continuous operator valued functions on the interval $[0, T]$.

Using the principle of uniform boundedness this space can be made into a Banach space with the norm

$$
\|F\|_{\infty}:=\sup _{0 \leq t \leq T}\|F(t)\|_{\mathcal{L}(X)} \quad \text { for } \quad F \in \mathcal{X} .
$$

On the space $\mathcal{X}$ we now define an abstract Volterra operator, and then show that its norm is less than 1 . We observe that for $F \in \mathcal{X}$ the operator

$$
D(A) \ni x \mapsto \int_{0}^{t} F(t-s) C(\tau) T(\tau) x d \tau
$$

is bounded in $X$ by (3.19). Thus it can be extended uniquely to a bounded operator on $X$ denoted by

$$
\left[V_{A}^{B} F\right](t):=\int_{0}^{t} F(t-s) C(\tau) T(\tau) d \tau \quad \text { for } F \in \mathcal{X} .
$$

Indeed, we have $V_{A}^{B}: \mathcal{X} \rightarrow \mathcal{X}$, since

$$
\left\|\left[V_{A}^{B} F\right](t)\right\|_{\mathcal{L}(X)} \leq \gamma\|F\|_{\infty}
$$


for $0 \leq t \leq T$, and since the mapping $t \mapsto\left[V_{A}^{B} F\right](t) x$ is continuous for $x \in D(A)$. This can be shown by the estimate

$$
\begin{aligned}
& \left\|\left[V_{A}^{B} F\right](t+h) x-\left[V_{A}^{B} F\right](t) x\right\| \\
& =\left\|\int_{0}^{t+h} F(t+h-s) C(s) T(s) x d s-\int_{0}^{t} F(t-s) C(s) T(s) x d s\right\| \\
& \leq \int_{0}^{t}\|(F(t+h-s)-F(t-s)) C(s) T(s) x\| d s \\
& \quad+\int_{t}^{t+h}\|F(t+h-s) C(s) T(s) x\| d s .
\end{aligned}
$$

For every $h>0$, the first term is bounded by the integrable function $s \mapsto 2\|F\|_{\infty}\|C(s) T(s) x\|$ and vanishes a. e. for $h \searrow 0$, while the second term is bounded by $h\|F\|_{\infty} \sup _{s \in[0, T]}\|C(s) T(s) x\|$. Thus for $h \searrow 0$ the whole expression vanishes. For $h \nearrow 0$ we argue analogously. Finally, it results from (3.25) that

$$
\left\|V_{A}^{B}\right\|_{\mathcal{L}(\mathcal{X})} \leq \gamma<1 .
$$

Second step: By the last norm estimate, the operator $\left(I d-V_{A}^{B}\right) \in \mathcal{L}(\mathcal{X})$ is invertible and

$$
\left(I d-V_{A}^{B}\right)^{-1}=\sum_{n=0}^{\infty}\left(V_{A}^{B}\right)^{n},
$$

where the right hand side converges with respect to the norm of $\mathcal{X}$.

Consider now the function $S T: t \mapsto S(t) T(t)$ in the space $\mathcal{X}$ and define a strongly continuous function

$$
t \mapsto U(t):=\left[\left(I d-V_{A}^{B}\right)^{-1} S T\right](t) .
$$

We will show that under the above additional assumptions the function $(U(t))_{t \in[0, T]}$ can be extended to a $C_{0}$-semigroup. First, we know that $(U(t))_{t \in[0, T]}$ is strongly continuous and satisfies the integral equation

$$
U(t) x=S(t) T(t) x+\int_{0}^{t} U(t-\tau) C(\tau) T(\tau) x d \tau
$$

for $x \in D(A)$ and $0 \leq t \leq T$. It is also given by a series expansion

$$
U(t)=\sum_{n=0}^{\infty}\left(\left(V_{A}^{B}\right)^{n} S T\right)(t) \quad \text { for } 0 \leq t \leq T .
$$

Third Step: To prove the semigroup property we will show that $U(\cdot)$ leaves the space $D$ invariant and solves the differential equation

$$
\frac{d}{d t} U(t) x=(A+B) U(t) x=U(t)(A+B) x
$$


for all $x \in D=D(A) \cap D(B)$.

In order to study the difference quotient $\frac{1}{h}(U(t+h)-U(t))$ we consider the terms of the series expansion

$$
\left(\left(V_{A}^{B}\right)^{n} S T\right)(t+h)-\left(\left(V_{A}^{B}\right)^{n} S T\right)(t) .
$$

By induction we will show that we can split these terms as

$$
\left(\left(V_{A}^{B}\right)^{n} S T\right)(t+h)-\left(\left(V_{A}^{B}\right)^{n} S T\right)(t)=\Delta_{h}^{1, n}(t)+\Delta_{h}^{2, n}(t)+\Delta_{h}^{3, n}(t),
$$

such that

and

$$
\frac{1}{h} \Delta_{h}^{1, n}(t) x \rightarrow B\left(\left(V_{A}^{B}\right)^{n} S T\right)(t) x
$$

$$
\frac{1}{h}\left(\Delta_{h}^{2, n}(t)+\Delta_{h}^{3, n+1}(t)\right) x \rightarrow A\left(\left(V_{A}^{B}\right)^{n} S T\right)(t) x
$$

for $h \searrow 0$ and $x \in D(A) \cap D(B)$. Together with the series expansion we then obtain

for all $x \in D(A) \cap D(B)$.

$$
\frac{d}{d t} U(t) x=(A+B) U(t) x
$$

We have for $n=0$ and $n=1$

$$
\begin{aligned}
& S(t+h) T(t+h) x-S(t) T(t) x \\
& =\underbrace{[S(t+h)-S(t)] T(t) x}_{=: \Delta_{h}^{1,0}(t) x}+\underbrace{S(t+h)[T(t+h)-T(t)] x}_{=: \Delta_{h}^{2,0}(t) x}+\underbrace{0}_{=: \Delta_{h}^{3,0}(t) x}
\end{aligned}
$$

and

$$
\begin{aligned}
& \left(\left(V_{A}^{B}\right) S T\right)(t+h) x-\left(\left(V_{A}^{B}\right) S T\right)(t) x \\
& =\int_{0}^{t+h} S T(t+h-\tau) C(\tau) T(\tau) x d \tau-\int_{0}^{t} S T(t-\tau) C(\tau) T(\tau) x d \tau \\
& =\underbrace{\int_{0}^{t}[S(t+h-\tau)-S(t-\tau)] T(t-\tau) C(\tau) T(\tau) x d \tau}_{=: \Delta_{h}^{1,1}(t) x} \\
& +\underbrace{\int_{0}^{t} S(t+h-\tau)[T(t+h-\tau)-T(t-\tau)] C(\tau) T(\tau) x d \tau}_{=: \Delta_{h}^{2,1}(t) x} \\
& +\underbrace{\int_{t}^{t+h} S(t+h-\tau) T(t+h-\tau) C(\tau) T(\tau) x d \tau}_{=: \Delta_{h}^{3,1}(t) x} .
\end{aligned}
$$

From this splitting we obtain

$$
\lim _{h \searrow 0} \frac{1}{h} \Delta_{h}^{1,0}(t) x=\lim _{h \searrow 0} \frac{1}{h}[S(t+h)-S(t)] T(t) x=B S(t) T(t) x,
$$


and

$$
\begin{aligned}
\lim _{h \searrow 0} & \frac{1}{h}\left(\Delta_{h}^{2,0}(t)+\Delta_{h}^{3,1}(t)\right) x \\
= & \lim _{h \searrow 0}\left(\frac{1}{h} S(t+h)[T(t+h)-T(t)] x\right. \\
& \left.\quad+\int_{t}^{t+h} S(t+h-\tau) T(t+h-\tau) C(\tau) T(\tau) x d \tau\right) \\
= & A S(t) T(t) x .
\end{aligned}
$$

Moreover,

$$
\begin{aligned}
\lim _{h \searrow 0} \frac{1}{h} \Delta_{h}^{1,1}(t) x & =\lim _{h \searrow 0} \frac{1}{h} \int_{0}^{t+h} B \int_{t-\tau}^{t+h-\tau} S(\sigma) d \sigma T(t-\tau) C(\tau) T(\tau) x d \tau \\
& =B\left(V_{A}^{B} S T\right)(t) x
\end{aligned}
$$

by the continuity of $T(t-\cdot) C(\cdot) T(\cdot) x$ in $D(B)$.

For the induction $(n \rightarrow n+1)$ let the claim be fulfilled for $n-1$ and $n$. Now, we calculate

$$
\begin{aligned}
& \left(\left(V_{A}^{B}\right)^{n+1} S T\right)(t+h) x-\left(\left(V_{A}^{B}\right)^{n+1} S T\right)(t) x \\
& =\int_{0}^{t+h}\left(\left(V_{A}^{B}\right)^{n} S T\right)(t+h-\tau) C(\tau) T(\tau) x d \tau \\
& -\int_{0}^{t}\left(\left(V_{A}^{B}\right)^{n} S T\right)(t-\tau) C(\tau) T(\tau) x d \tau \\
& =\int_{0}^{t}\left[\Delta_{h}^{1, n}(t-\tau)+\Delta_{h}^{2, n}(t-\tau)+\Delta_{h}^{3, n}(t-\tau)\right] C(\tau) T(\tau) x d \tau \\
& +\int_{t}^{t+h}\left[\left(V_{A}^{B}\right)^{n} S T\right](t+h-\tau) C(\tau) T(\tau) x d \tau \\
& =\underbrace{\int_{0}^{t} \Delta_{h}^{1, n}(t-\tau) C(\tau) T(\tau) x d \tau}_{=: \Delta_{h}^{1, n+1}(t) x}+\underbrace{\int_{0}^{t} \Delta_{h}^{2, n}(t-\tau) C(\tau) T(\tau) x d \tau}_{=: \Delta_{h}^{2, n+1}(t) x} \\
& +\int_{0}^{t} \Delta_{h}^{3, n}(t-\tau) C(\tau) T(\tau) x d \tau \\
& +\int_{t}^{t+h}\left[\left(V_{A}^{B}\right)^{n} S T\right](t+h-\tau) C(\tau) T(\tau) x d \tau,
\end{aligned}
$$


where the sum of the two last integrals equals $\Delta_{h}^{3, n+1}(t) x$. By induction we obtain

$$
\begin{aligned}
& \lim _{h \searrow 0} \frac{1}{h} \Delta_{h}^{1, n+1}(t) x=\lim _{h \searrow 0} \frac{1}{h} \int_{0}^{t} \Delta_{h}^{1, n}(t-\tau) C(\tau) T(\tau) x d \tau \\
& \quad=\int_{0}^{t} B\left(\left(V_{A}^{B}\right)^{n} S T\right)(t-\tau) C(\tau) T(\tau) x d \tau=B\left(\left(V_{A}^{B}\right)^{n+1} S T\right)(t) x
\end{aligned}
$$

and

$$
\begin{aligned}
\lim _{h \searrow 0} & \frac{1}{h}\left(\Delta_{h}^{2, n}(t) x+\Delta_{h}^{3, n+1}(t) x\right) \\
= & \lim _{h \searrow 0} \frac{1}{h} \int_{0}^{t}\left(\Delta_{h}^{2, n-1}(t-\tau)+\Delta_{h}^{3, n}(t-\tau)\right) C(\tau) T(\tau) x d \tau \\
& \quad+\lim _{h \searrow 0} \frac{1}{h} \int_{t}^{t+h}\left(\left(V_{A}^{B}\right)^{n-1} S T\right)(t+h-\tau) C(\tau) T(\tau) x d \tau \\
= & A \int_{0}^{t}\left(\left(V_{A}^{B}\right)^{n-1} S T\right)(t-\tau) C(\tau) T(\tau) x d \tau+0 \\
= & A\left(\left(V_{A}^{B}\right)^{n} S T\right)(t) x,
\end{aligned}
$$

since $\left.\left(V_{A}^{B}\right)^{n-1} S T\right)(0)=0$ for $n \geq 2$.

To justify the last claim we consider first the limit

$$
\begin{aligned}
\lim _{h \searrow 0} \quad & \frac{1}{h} \int_{0}^{t-\varepsilon}\left(\Delta_{h}^{2, n-1}(t-\tau)+\Delta_{h}^{3, n}(t-\tau)\right) C(\tau) T(\tau) x d \tau \\
& =A \int_{0}^{t-\varepsilon}\left(\left(V_{A}^{B}\right)^{n-1} S T\right)(t-\tau) C(\tau) T(\tau) x d \tau
\end{aligned}
$$

for $x \in D$ and small $\varepsilon>0$ and use then the assumptions (3.23) and (3.24). The second half of the differential equation (3.27) is proved by an analogous induction after the following rearrangement. By an integration by parts we 
obtain for the integral term

$$
\begin{aligned}
\int_{0}^{t} S(t-\tau) T(t-\tau) C(\tau) T(\tau) x d \tau \\
=\quad \int_{0}^{t} S(t-\tau) T(t-\tau) A S(\tau) T(\tau) x d \tau-[S(t-s) T(t-s) S(s) T(s) x]_{0}^{t} \\
\quad+\int_{0}^{t} S(t-\tau) T(t-\tau) B S(\tau) T(\tau) x d \tau \\
\quad-\int_{0}^{t} S(t-\tau) T(t-\tau) A S(\tau) T(\tau) x d \tau \\
\quad-\int_{0}^{t} S(t-\tau) B T(t-\tau) S(\tau) T(\tau) x d \tau \\
=\quad \int_{0}^{t} S(\tau) T(\tau) B S(t-\tau) T(t-\tau) x d \tau \\
\quad-\int_{0}^{t} S(\tau) B T(\tau) S(t-\tau) T(t-\tau) x d \tau \\
\quad \int_{0}^{t} S(\tau)(T(\tau) B-B T(\tau)) S(t-\tau) T(t-\tau) x d \tau
\end{aligned}
$$

for all $x \in D(A) \cap D(B)$ and we can argue by an analogous induction as above.

The semigroup property of $(U(t))_{t \geq 0}$ now follows from (3.27) by differentiating the function $s \mapsto U(t-s) U(s) x$ for all $x \in D(A) \cap D(B)$.

Problem 3.3. Prove the semigroup property of $(U(t))_{t \geq 0}$ directly (cf. [17], Lemma 1.4) by using the series expansion (3.21). Remove the assumption of $(T(t))$-invariance of $D(B)$ and the other regularity assumptions.

With the aid of the preceding lemma we can now prove wellposedness in the parabolic case. Some technical steps are anticipated in the following two lemmas (cf. [12], Lemma 5.6.2, Section 5.III, and Lemma 5.6.4). For the sake of simplicity, we denote by $c$ a generic constant.

Lemma 3.4. Let $(A(t), D)_{t \in \mathbb{R}}$ be a family of linear, densely defined operators on some Banach space $X$ fulfilling the conditions in Assumption 3.1. Then the following estimates hold:

$$
\begin{aligned}
& \text { (3.28) }\left\|\left(A\left(t_{1}\right)-A\left(t_{2}\right)\right) e^{\tau A(s)}\right\| \leq \frac{c}{\tau}\left|t_{1}-t_{2}\right|^{\alpha} \text { for } t_{1}, t_{2}, s \in \mathbb{R}, \tau>0 . \\
& \text { (3.29) }\left\|A\left(t_{1}\right) e^{\tau A\left(t_{2}\right)}\right\| \leq \frac{c}{\tau} \text { for } t_{1}, t_{2} \in \mathbb{R}, \tau>0 . \\
& \text { (3.30) }\left\|A(s)\left[e^{\tau A\left(t_{1}\right)}-e^{\tau A\left(t_{2}\right)}\right]\right\| \leq \frac{c}{\tau}\left|t_{1}-t_{2}\right|^{\alpha} \text { for } t_{1}, t_{2}, s \in \mathbb{R}, \tau>0 . \\
& \text { (3.31) }\left\|A\left(t_{1}\right) e^{\tau A\left(t_{1}\right)}-A\left(t_{2}\right) e^{\tau A\left(t_{2}\right)}\right\| \leq \frac{c}{\tau}\left|t_{1}-t_{2}\right|^{\alpha} \text { for } t_{1}, t_{2} \in \mathbb{R}, \tau>0 .
\end{aligned}
$$


If the function $t \mapsto A(t) y$ is continuously differentiable for all $y \in D$, we obtain that the function $s \mapsto e^{\tau A(s)} x$ is continuously differentiable in $\tau>0$ for all $x \in X$ as well.

Lemma 3.5. Let $(A(t), D)_{t \in \mathbb{R}}$ be a family of linear, densely defined operators on some Banach space $X$ fulfilling the conditions in Assumption 3.1. Denote by

$$
R_{1}(t, s):=[A(t)-A(s)] e^{(t-s) A(s)} .
$$

Then for every $0<\beta \leq \alpha$, there is a constant $c_{\beta}$ such that

$$
\left\|R_{1}(t, s)-R_{1}(\tau, s)\right\| \leq c_{\beta}(t-\tau)^{\beta}(\tau-s)^{\alpha-\beta-1}
$$

for $s<\tau<t \in \mathbb{R}$.

Theorem 3.6. Let $(A(t), D)_{t \in \mathbb{R}}$ be a family of linear, densely defined operators on some Banach space $X$ fulfilling the conditions in Assumption 3.1. Then there exists a unique evolution semigroup $(\mathcal{T}(t))_{t \geq 0}$ on the space $\mathcal{C}_{0}$ with generator $(\mathcal{G}, D(\mathcal{G}))$ such that $\mathcal{C}^{1} \cap C_{0}(\mathbb{R}, D) \subset D(\overline{\mathcal{G}})$ and

$$
\mathcal{G} f+f^{\prime}=A(\cdot) f
$$

for all $f \in \mathcal{C}^{1} \cap C_{0}(\mathbb{R}, D)$. Moreover, we have $\mathcal{T}(t): \mathcal{C}_{0} \rightarrow C_{0}(\mathbb{R}, D)$ for $t>0$.

Together with the characterization of wellposedness in Theorem 2.9 we obtain the following corollary.

Corollary 3.7 (Parabolic case). In the situation of Theorem 3.6, there exists a unique exponentially bounded evolution family $(U(t, s))_{t \geq s}$ of bounded operators on $X$ solving $(N C P)$. Thus, the nonautonomous Cauchy problem $(N C P)$ is wellposed and for every $u_{s} \in D$ there is a unique strict solution $t \mapsto U(t, s) u_{s}$. Moreover, for every $u_{s} \in X$ the function $t \mapsto U(t, s) u_{s}$ is a classical solution of $(N C P)$ for $s<t$.

Proof of Theorem 3.6. First Step: We consider the $C_{0}$-semigroup $\left(e^{t A(\cdot)}\right)_{t \geq 0}$ and the translation group $(\mathcal{T} r(t))_{t \in \mathbb{R}}$ on the space $\mathcal{C}_{0}$. The generator of $\left(e^{t A(\cdot)}\right)_{t \geq 0}$ is $(A(\cdot), \mathcal{D})$, where the domain $\mathcal{D}$ is given by

$$
\mathcal{D}:=\left\{f \in \mathcal{C}_{0}: f(s) \in D \text { for all } s \in \mathbb{R} \text { and } s \mapsto A(s) f(s) \in \mathcal{C}_{0}\right\} .
$$

Since we assume constant domains, Assumption $3.1(P 1)$, we see that $\mathcal{T} r(t) \mathcal{D}$ $\subset \mathcal{D}$.

Moreover, for all $f \in \mathcal{D}$ we obtain from the Hölder condition, Assumption 3.1 (P3), and equation (3.29) that

$$
\int_{0}^{t}\left\|[A(\cdot)-A(\cdot-\tau)] \mathcal{T} r(\tau) e^{\tau A(\cdot)} f\right\| d \tau \leq \int_{0}^{t} c \tau^{\alpha-1} d \tau\|f\|=\frac{c t^{\alpha}}{\alpha}\|f\| .
$$


Choosing $T>0$ small enough, we obtain

$$
\int_{0}^{T}\left\|[A(\cdot)-A(\cdot-\tau)] \mathcal{T} r(\tau) e^{\tau A(\cdot)} f\right\| d \tau \leq \gamma\|f\|
$$

with $\gamma<1$ and thus all conditions of the first part in Lemma 3.2 are satisfied. In fact, the Hölder condition even implies that the operator

$$
(V F)(t):=\int_{0}^{t} F(t-\tau)[A(\cdot)-A(\cdot-\tau)] \mathcal{T} r(\tau) e^{\tau A(\cdot)} d \tau
$$

defined on the Banach space $\mathcal{X}:=C\left([0, T], \mathcal{L}_{s}\left(\mathcal{C}_{0}\right)\right)$ of all strongly continuous operator valued functions on the interval $[0, T]$ has zero spectral radius. By induction we prove that

$$
\left\|\left[(V)^{n} F\right](t)\right\| \leq \frac{C^{n} \Gamma(\alpha)^{n}}{\Gamma(n \alpha)} t^{n \alpha-1}\|F\|_{\infty}
$$

for $n \in \mathbb{N}, t \geq 0$, where $\Gamma$ denotes the Gamma-function. The case $n=1$ has been proven in the first step. For the induction $(n \rightarrow n+1)$ we calculate for $x \in D$ with $\|x\|=1$

$$
\begin{aligned}
\left\|\left([V]^{n+1} F\right)(t) x\right\| & =\left\|\int_{0}^{t}\left([V]^{n} F\right)(t-r)[A(\cdot)-A(\cdot-r)] e^{r A(\cdot)} x d r\right\| \\
& \stackrel{(3.28)}{\leq} \int_{0}^{t} C r^{\alpha-1}\left\|\left(V^{n} F\right)(t-r)\right\|\|x\| d r \\
& \stackrel{\text { ind }}{\leq} \int_{0}^{t} C^{n+1} \frac{\Gamma(\alpha)^{n}}{\Gamma(n \alpha)}(t-r)^{n \alpha-1} r^{\alpha-1}\|F\|_{\infty} d r \\
& =\frac{C^{n+1} \Gamma(\alpha)^{n+1}}{\Gamma((n+1) \alpha)} t^{n \alpha-1}\|F\|_{\infty} .
\end{aligned}
$$

Here, we used the identity

$$
\int_{0}^{t}(t-r)^{\beta-1} r^{\gamma-1} d r=\frac{\Gamma(\beta) \Gamma(\gamma)}{\Gamma(\beta+\gamma)} t^{\beta+\gamma-1}, \quad \text { for } \beta, \gamma>0 .
$$

Second Step: By Lemma 3.2 we obtain a strongly continuous function $(\mathcal{T}(t))_{t \geq 0}$ solving the integral equation

$$
\begin{aligned}
\mathcal{T}(t)= & \mathcal{T} r(t) e^{t A(\cdot)} \\
& +\int_{0}^{t} \mathcal{T}(t-\tau)[A(\cdot)-A(\cdot-\tau)] \mathcal{T} r(\tau) e^{\tau A(\cdot)} d \tau \\
= & \mathcal{T} r(t) e^{t A(\cdot)} \\
& +\int_{0}^{t} \mathcal{T}(\tau)[A(\cdot)-A(\cdot+t-\tau)] \mathcal{T} r(t-\tau) e^{(t-\tau) A(\cdot)} d \tau .
\end{aligned}
$$


It is also given by the series expansion

$$
\mathcal{T}(t)=\sum_{n=0}^{\infty} V^{n} \mathcal{T} r(t) e^{t A(\cdot)}
$$

In the next step we show that it is an evolution semigroup and that for the generator of $(\mathcal{T}(t))_{t \geq 0}$, denoted by $(\mathcal{G}, D(\mathcal{G}))$, we obtain $\mathcal{D} \cap \mathcal{C}^{1} \subseteq D(\mathcal{G})$ and

$$
\mathcal{G} f=-f^{\prime}+A(\cdot) f \quad \text { for } f \in \mathcal{D} \cap \mathcal{C}^{1} .
$$

Third Step: We assume now $(A(t))_{t \in \mathbb{R}}$ to be strongly continuously differentiable on $D$. Then, invariance of the domain $\mathcal{C}^{1}$ under the semigroup $\left(e^{t A(\cdot)}\right)_{t \geq 0}$ and property (3.22) follow from the second part of Lemma 3.4 and the assumed differentiability of $t \mapsto A(t) y$ for $y \in D$. From the analyticity of $\left(e^{t A(\cdot)}\right)_{t \geq 0}$ we infer $e^{t A(\cdot)} f \in \mathcal{D}$ for all $f \in \mathcal{C}_{0}$ and $t>0$. Finally, the properties (3.23) and (3.24) follow from the considerations below. By the second part of Lemma 3.2 we infer that the dense subspace $\mathcal{D} \cap \mathcal{C}^{1}$ is invariant under $\mathcal{T}(t)$, thus a core for the generator. The representation (3.36) is then given on an invariant core of $\mathcal{G}$ and by Lemma 1.3 we see that $(\mathcal{T}(t))_{t \geq 0}$ is in fact an evolution semigroup and, at the same moment, that $(N C P)$ is wellposed.

We will even prove that $\mathcal{T}(t): \mathcal{C}_{0} \rightarrow \mathcal{D}$ for $t>0$, which implies the existence of unique classical solutions of $(N C P)$ for all initial values $x_{s} \in X$ (see Proposition 2.10). This can be seen using the series representation (3.35) of $(\mathcal{T}(t))_{t \geq 0}$. We will show that this series also converges for $t>0$ in the $\mathcal{L}\left(\mathcal{C}_{0}, \mathcal{D}\right)$-norm, where we define a Banach space norm $\|\cdot\|_{\mathcal{D}}$ on $\mathcal{D}$ by

$$
\|f\|_{\mathcal{D}}:=\sup _{s \in \mathbb{R}}\|A(s) f(s)\|
$$

for $f \in \mathcal{D}$. Consider the terms of the expansion. For the 0 -th term we obtain by (3.29) in Lemma 3.4 that

$$
\left\|\mathcal{T} r(t) e^{t A(\cdot)}\right\|_{\mathcal{L}\left(\mathcal{C}_{0}, \mathcal{D}\right)} \leq \frac{c}{t}
$$


for $t>0$. To estimate the first term of the expansion we use (3.28), (3.29), (3.30), and (3.31) which yields

$$
\begin{aligned}
\| A(s+ & t) \int_{0}^{t} e^{(t-\tau) A(s+\tau)}[A(s+\tau)-A(s)] e^{(\tau) A(s)} d \tau \| \\
\leq \quad & \int_{0}^{t}\left\|[A(s+t)-A(s+\tau)] e^{(t-\tau) A(s+\tau)} R_{1}(s+\tau, s)\right\| d \tau \\
& +\left\|\int_{0}^{t} A(s+\tau) e^{(t-\tau) A(s+\tau)} R_{1}(s+\tau, s) d \tau\right\| \\
\leq \quad & \int_{0}^{t}(t-\tau)^{\alpha-1} \tau^{\alpha-1} d \tau \\
& +\int_{0}^{t}\left\|\left[A(s+t) e^{(t-\tau) A(s+t)}-A(s+\tau) e^{(t-\tau) A(s+\tau)}\right] R_{1}(s+\tau, s)\right\| d \tau \\
& +\int_{0}^{t}\left\|A(s+t) e^{(t-\tau) A(s+t)}\left[R_{1}(s+t, s)-R_{1}(s+\tau, s)\right]\right\| d \tau \\
+ & \left\|\int_{0}^{t} A(s+t) e^{(t-\tau) A(s+t)} d \tau R_{1}(s+t, s)\right\| \\
\leq \quad & \int_{0}^{t}(t-\tau)^{\alpha-1} \tau^{\alpha-1} d \tau+\int_{0}^{t} c_{T}(t-\tau)^{\alpha-1} \tau^{\alpha-1} d \tau \\
+ & \int_{0}^{t} c_{T}(t-\tau)^{\beta-1} \tau^{\alpha-\beta-1} d \tau+c_{T} t^{\alpha-1}
\end{aligned}
$$

for all $s \in \mathbb{R}$ and $T \geq t>0$. Thus, we obtain the estimate

$$
\left\|A(s+t) \int_{0}^{t} e^{(t-\tau) A(s+\tau)}[A(s+\tau)-A(s)] e^{(\tau) A(s)} d \tau\right\| \leq c_{T} t^{\alpha-1},
$$

which implies

$$
\left\|\int_{0}^{t} \mathcal{T} r(t-\tau) e^{(t-\tau) A(\cdot)}[A(\cdot)-A(\cdot-\tau)] \mathcal{T} r(\tau) e^{\tau A(\cdot)}\right\|_{\mathcal{L}\left(\mathcal{C}_{0}, \mathcal{D}\right)} \leq \frac{c_{T}}{t} t^{\alpha}
$$

By an induction, similar to the one above, we obtain the desired convergence in $\mathcal{L}\left(\mathcal{C}_{0}, \mathcal{D}\right)$.

Last step: The general case in which $(A(t))_{t \in \mathbb{R}}$ is not necessarily strongly continuously differentiable follows by an approximation argument as in [12], Section 5.III. 
Remark 3.8. Acquistapace [1] discusses several starting points for proving wellposedness in the parabolic case. Reasonable approximations for the solving evolution family $(U(t, s))_{t \geq s}$ could be

$$
\begin{aligned}
& V_{1}(t, s):=e^{(t-s) A(s)} \\
& V_{2}(t, s):=e^{(t-s) A(t)} \\
& V_{3}(t, s):=e^{(t-s) A(T)} \\
& V_{4}(t, s):=e^{\int_{s}^{t} A(\tau) d \tau} .
\end{aligned}
$$

The first was chosen in our approach. The second leads, by an analogous formal calculation differentiating $\tau \mapsto U(t, \tau) e^{(\tau-s) A(\tau)}$, to the integral equation

$$
U(t, s)=e^{(t-s) A(t)}+\int_{s}^{t} U(t, \tau)[(\tau-s) \dot{A}(\tau)] e^{(\tau-s) A(\tau)} d \tau .
$$

Rewritten in terms of the evolution semigroup we obtain

$$
\mathcal{T}(t)=e^{t A(\cdot)} \mathcal{T} r(t)+\int_{0}^{t} \mathcal{T}(t-\tau)[\tau \dot{A}(\cdot)] e^{\tau A(\cdot)} \mathcal{T} r(\tau) d \tau
$$

Regarding the formal identity

$$
\tau \dot{A}(x) e^{\tau A(x)}=\frac{d}{d x} e^{\tau A(x)}-e^{\tau A(x)} \frac{d}{d x},
$$

we end up with a similar perturbation problem to that considered in Lemma 3.2.

Final Remark 3.9. Our method is based on the idea to use Hille-Yosida type generation theorems to obtain wellposedness for nonautonomous Cauchy problems. In addition to being a unifying approach it also yields to new wellposedness results (e.g., see [14]).

Acknowledgment. The author wishes to thank Rainer Nagel and Abdelaziz Rhandi for many helpful comments.

\section{REFERENCES}

[1] P. Acquistapace, Abstract linear nonautonomous parabolic equations: a survey, in "Differential Equations in Banach Spaces", eds. G. Dore et al, Marcel Dekker, New York, 1993.

[2] D. E. Evans, Time dependent perturbations and scattering of strongly continuous groups on Banach spaces, Math. Ann. 221 (1976), 275-290.

[3] J. Goldstein, On the absence of necessary conditions for linear evolution operators, Proc. Amer. Math. Soc. 64 (1977), 77-80.

[4] T. Graser, Operator multipliers generating strongly continuous semigroups, Semigroup Forum, to appear.

[5] J. S. Howland, Stationary scattering theory for time-dependent Hamiltonians, Math. Ann. 207 (1974), 315-335.

[6] H. Kellermann, Linear evolution equations with time-dependent domain, Semesterbericht Funktionalanalysis, Tübingen, WS 1985. 
[7] Y. Latushkin, S. Montgomery-Smith and T. Randolph, Evolutionary semigroups and dichotomies of linear skew-product flows on locally compact spaces with Banach fibers, J. Differential Equations, to appear.

[8] Y. Latushkin and T. W. Randolph, Dichotomy of differential equations on Banach spaces and an algebra of weighted translation operators, Integral Equations Operator Theory, 23, (1995), 472-500.

[9] R. Nagel, Semigroup methods for non-autonomous Cauchy problems, in "Evolution Equations", eds. G. Ferreyra et al, Lect. Notes Pure Appl. Math., \#168, Marcel Dekker, New York, 1995, 301-316.

[10] R. Nagel and A. Rhandi, A characterization of Lipschitz continuous evolution families on Banach spaces, "Operator Theory: Advances and Application", \#75, Birkhäuser Verlag, Basel, 1995, 275-288.

[11] G. Nickel, On evolution semigroups and wellposedness of nonautonomous Cauchy problems, Dissertation, Tübingen, 1996.

[12] A. Pazy, Semigroups of linear operators and applications to partial differential equations, Springer-Verlag, Berlin, 1983.

[13] F. Räbiger, A. Rhandi and R. Schnaubelt, Perturbation and an abstract characterization of evolution semigroups, J. Math. Anal. Appl. 198 (1996), 516-533.

[14] F. Räbiger, A. Rhandi and R. Schnaubelt, Non-autonomous Miyadera Perturbation. Ulmer Seminare 1 (1996), 331-357.

[15] F. Räbiger and R. Schnaubelt, The spectral mapping theorem for evolution semigroups on spaces of vector valued functions, Semigroup Forum, 52 (1996), 225-239.

[16] R. T. Rau, Hyperbolic evolution semigroups on vector valued function spaces, Semigroup Forum 48 (1994), 107-118.

[17] A. Rhandi, Dyson-Phillips expansion and unbounded perturbations of linear $C_{0}-$ semigroups, J. Comp. Appl. Math. 44 (1992), 339-349.

[18] A. Rhandi, Lipschitz-stetige evolutionsfamilien und die exponentielle dichotomie, Dissertation, Tübingen 1994.

Arbeitsbereich Funktionalanalysis

Mathematisches Institut

UNIVERSITÄT TÜBINGEN

Auf Der Morgenstelle 10

D - 72076 TÜBINGEN, GERMANY

E-mail address: grni@michelangelo.mathematik.uni-tuebingen.de 


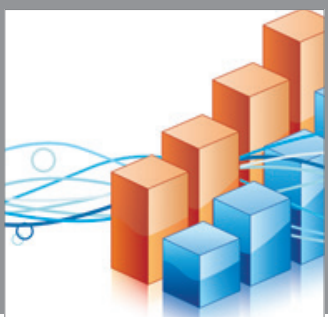

Advances in

Operations Research

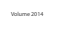

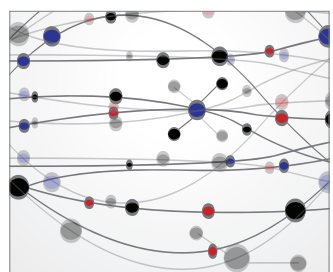

\section{The Scientific} World Journal
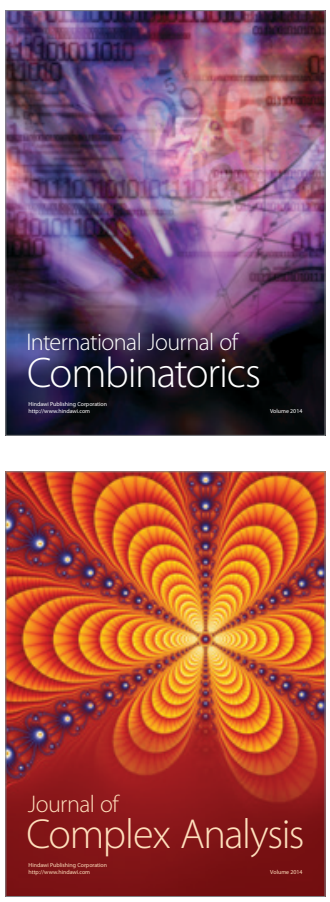

International Journal of

Mathematics and

Mathematical

Sciences
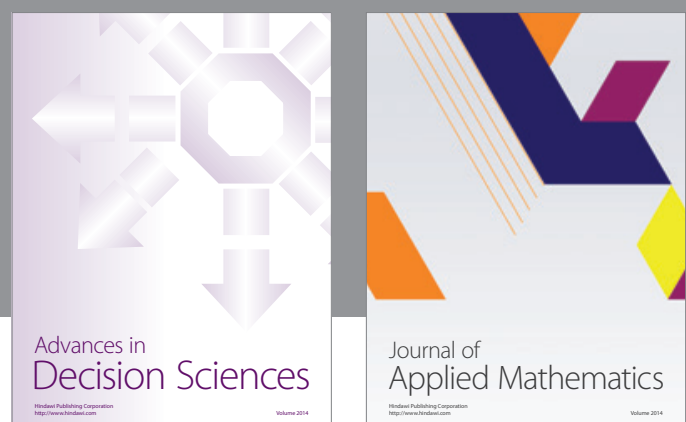

Journal of

Applied Mathematics
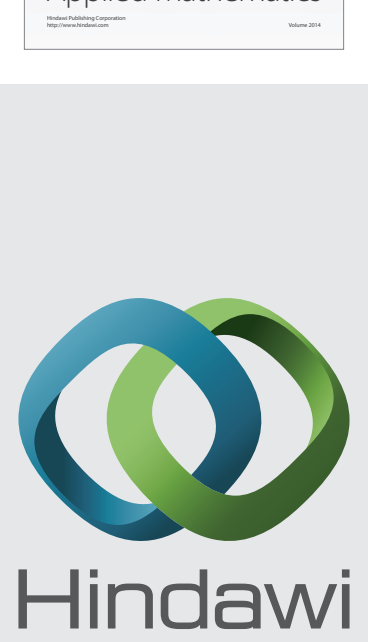

Submit your manuscripts at http://www.hindawi.com
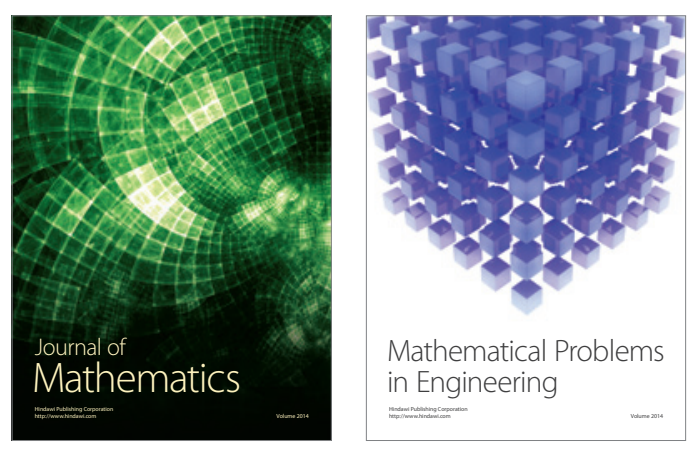

Mathematical Problems in Engineering
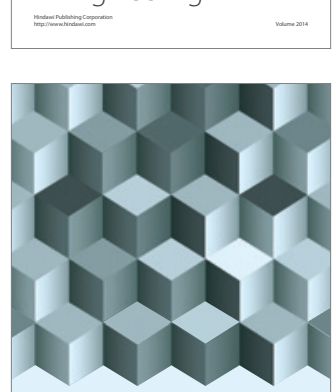

Journal of

Function Spaces
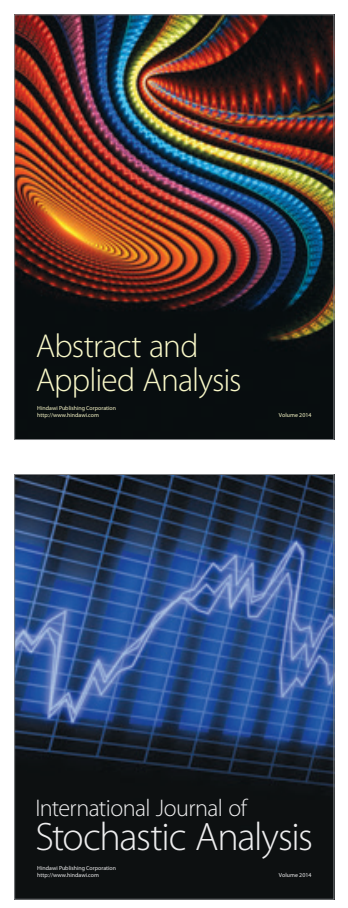

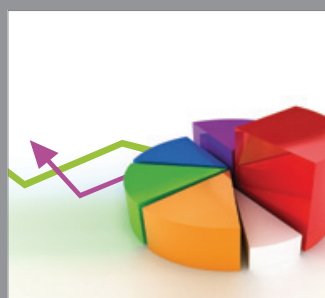

ournal of

Probability and Statistics

Promensencen
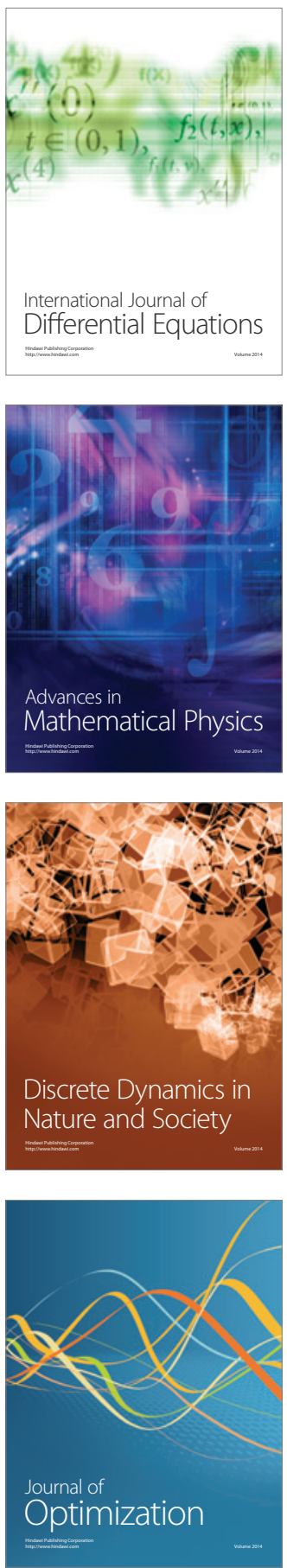